\title{
Integration of Topological Constraints in Medical Image Segmentation
}

\author{
F. Ségonne and B. Fischl ${ }^{1}$
}

\begin{abstract}
Topology is a strong global constraint that can be useful in generating geometrically accurate segmentations of anatomical structures. Conversely, topological "defects" or departures from the true topology of a structure due to segmentation errors can greatly reduce the utility of anatomical models. In this chapter we cover methods for integrating topological constraints into segmentation procedures in order to generate geometrically accurate and topologically correct models, which is critical for many clinical and research applications.
\end{abstract}

\section{Introduction}

\subsection{Description of the Problem}

In medical imaging, the overall shape of a region of interest is typically prescribed by medical knowledge; it is usually known a priori. Excluding pathological cases, the shape of most macroscopic brain structures can be continuously deformed into a sphere. In mathematical terms, these structures have the topology of a sphere. Particularly, this implies that most brain structures consist of one single connected object that does not possess any handles (i.e. holes) or cavities. This is the case for noncortical structures (such as left and right ventricle, putamen, pallidum, amygdala, hippocampus, thalamus, and caudate nucleus - see Fig. 1-a), but it also holds for the cortex under some specific conditions ${ }^{2}$. In addition to their individual topological properties, anatomical structures occur in a charac-

\footnotetext{
${ }^{1}$ Support for this research was provided in part by the National Center for Research Resources (P41-RR14075, R01 RR16594-01A1 and the NCRR BIRN Morphometric Project BIRN002, U24 RR021382), the National Institute for Biomedical Imaging and Bioengineering (R01 EB001550, R01EB006758), the National Institute for Neurological Disorders and Stroke (R01 NS052585-01) as well as the Mental Illness and Neuroscience Discovery (MIND) Institute, and is part of the National Alliance for Medical Image Computing (NAMIC), funded by the National Institutes of Health through the NIH Roadmap for Medical Research, Grant U54 EB005149.

${ }^{2}$ The human cerebral cortex is a highly folded ribbon of gray matter (GM) that lies inside the cerebrospinal fluid (CSF) and outside the white matter (WM) of the brain.
} 


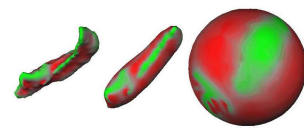

a)

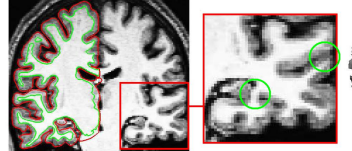

c)

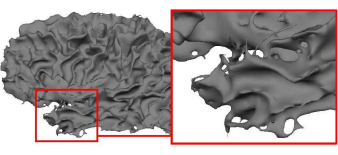

d)

e)

FIGURE 1. a) Subcortical structures have a spherical topology. For instance, the shape of the hippocampus can be continuously deformed onto a sphere. b) The human cerebral cortex is a highly folded ribbon of gray matter that lies inside the cerebrospinal fluid (the red interface) and outside the white matter of the brain (the green interface). When the midline connections between the left and right hemisphere are artificially closed, these two surfaces have the topology of a sphere. c) Due to the partial volume effect, subject motion, etc..., it becomes hard to distinguish opposite banks of a the gray matter. d) Segmentation algorithms that do not constrain the topology often produce cortical segmentations with several topological defects (i.e. handles, cavities, disconnected components). e) A close-up of a topologically-incorrect cortical surface representation.

teristic spatial pattern relative to one another (e.g. in the human brain, the amygdala is anterior and superior to the hippocampus). The set of individual topological properties and specific relationships between anatomical structures determine the global topology of a region of interest.

Although many clinical and research applications require accurate segmentations that respect the true anatomy of the targeted structures, only a few techniques have been proposed to achieve accurate and topologicallycorrect segmentations.

\subsection{Motivation}

Many neurodegenerative disorders, psychiatric disorders, and healthy aging are frequently associated with structural changes in the brain. These changes, which can cause alterations in the imaging properties of the brain tissue, as well as in morphometric properties of brain structures, can be captured and detected by sophisticated segmentation techniques. Certain clinical and research applications depend crucially on the accuracy and correctness of the representations (visualization $[11,12,56]$, spherical coordinate system and surface-based atlases $[12,16,17,18,22,50,56]$, shape analysis [16, 20, 31, 43, 53, 54], surface-based processing of functional data [12], and inter-subject registration [22, 51, 55], among others). Small geometric errors in a segmentation can easily change the apparent connectivity of the segmented structure, posing a problem to studies that aim at analyzing

Locally, its intrinsic "unfolded" structure is that of a 2D sheet, several millimeters thick. In the absence of pathology and assuming that the midline hemispheric connections are artificially closed, each cortical hemisphere can be considered as a simply-connected 2D sheet of neurons that carries the simple topology of a sphere - see Fig. 1-b 
the connectedness of different regions (e.g. dramatic underestimation of true geodesic distances). The accuracy and correctness of the representations can be critical factors in the success of studies investigating the subtle early effects of various disease processes.

\subsection{Challenges}

Accurate segmentation under anatomical consistency is challenging. Segmentation algorithms, which operate on the intensity or texture variations of the image, are sensitive to the artifacts produced by the image acquisition process. These limitations include image noise, low frequency image intensity variations or non-uniformity due to radio frequency (RF) inhomogeneities, geometric distortions in the images, partial volume averaging effect, and subject motion. Segmentation techniques that do not integrate any topological constraints generate segmentations that often contain deviations from the true topology of the structures of interest. These deviations are called topological defects and can be of three types: cavities, disconnected components, or handles (which are topologically equivalent to holes) that incorrectly connect parts of the volumes.

The integration of topological constraints significantly increases the complexity of the task. Topology is both a global and a local concept; small and local modifications of a geometric shape can change its global connectivity. Furthermore, topology is intrinsically a continuous concept (sec. 2.1) and topological notions are difficult to adapt into a discrete framework (sec. 2.2). Due to these difficulties, the set of techniques applicable to the segmentation of images is quite limited (sec. 3).

In this chapter, we present background material of central importance for the segmentation of medical images under topological constraints. We introduce some elementary notions of topology (sec. 2.1) and show how these notions can be adapted into a discrete framework and applied to the segmentation problem (sec. 2.2). Next, we describe the current state-ofthe-art segmentation of images under topological constraints (sec. 3) and detail the limitations of existing approaches (sec. 3). Section 4 concludes.

\section{Topology in Medical Imaging}

In medical image segmentation, one is interested in locating (accurately and also under topological constraints) specific regions of interest that are formed by one or several anatomical structures. Those can be represented equivalently by their volume or their surface and these two equivalent representations correspond to the two most common data structures used in medical imaging: 3D voxel grids and surfaces (such as triangulations or levelsets). 


\subsection{General Notions of Topology}

Topology is a branch of mathematics that studies the properties of geometric figures by abstracting their inherent connectivity while ignoring their detailed form. The exact geometry of the objects, their location and the details of their shape are irrelevant to the study of their topological properties. Schematically, this amounts to characterizing a geometric object (i.e. a surface or a volume) by its number of disconnected components, holes and cavities, but not by their position. For instance, the surface of a coffee mug with a handle has the same topology as the surface of a doughnut (this type of surface is called a one-handled torus).

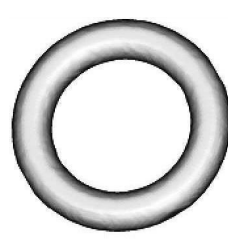

a)

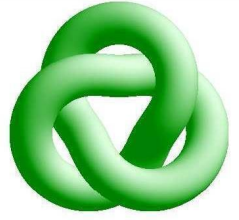

b)

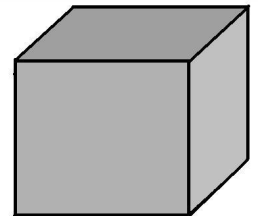

c)

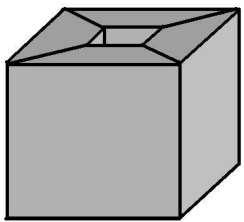

d)

FIGURE 2. a-b) Two tori that are homeomorphically equivalent. They share the same intrinsic topology. However, they do not share the same homotopy type as one cannot be continuously transformed into the other. c) A geometric object with a spherical topology; its Euler-characteristic is $\chi=v-e+f=8-12+6=2$. d) A geometric object with a toroidal topology and an Euler-characteristic of $\chi=v-e+f=16-32+16=0$.

\section{A - Homeomorphism, Genus, and Euler-Characteristic}

In medical imaging, the geometric entities under consideration are anatomical structures, which can frequently be advantageously represented by their surfaces. The Gauss-Bonnet theorem in differential geometry links the geometry of surfaces with their topology. Any compact connected orientable surface is homeomorphic to a sphere with some number of handles. This number of handles is a topological invariant called the genus. For example, a sphere is of genus 0 and a torus is of genus 1 . The genus $g$ is directly related to another topological invariant called the Euler-characteristic $\chi$ by the formula $\chi=2(1-g)^{3}$. The Euler-characteristic is of great practical interest because it can be calculated from any polyhedral decomposition (e.g. triangulation) of the surface by the simple formula $\chi=v-e+f$, where $v, e$ and $f$ denote respectively the number of vertices, edges and faces of the polyhedron. The Euler-characteristic of a sphere is $\chi=2$. This implies that any surface with $\chi=2$ is topologically equivalent (i.e. homeomorphic) to a sphere and therefore does not contain any handles. Surfaces with an Euler-characteristic $\chi<2$ have a topology that is different from that of a

\footnotetext{
${ }^{3}$ In the case of multiple surfaces involving $K$ connected components, the total genus is related to the total Euler-characteristic by the formula: $\chi=2(K-g)$.
} 
sphere. Note, however, that the Euler-characteristic does not provide any information about the localization of the handles.

$B$ - Intrinsic Topology and Homotopy Type

Homeomorphisms are used to define the intrinsic topology of an object, independently of the embedding space. The topological invariance of the Euler characteristic implies that the way a surface is decomposed (i.e. tessellated) does not influence its (intrinsic) topology. Any polyhedral decomposition of a surface will encode the same intrinsic topology. For example, a knotted solid torus has the same genus (and the same Euler-characteristic $\chi=0$ ) as a simple torus. In order to topologically differentiate surfaces, one needs a theory that considers the embedding space. Homotopy, which defines two surfaces to be homotopic if one can be continuously transformed into the other, is such a theory that provides a measure of an object's topology (see [8] for an excellent course in algebraic topology). We stress the fact that the Euler-characteristic does not define the homotopy type of a surface, since the embedding space is being ignored. In particular, this implies that a discrete representation of a surface using a polygonal decomposition with the desired Euler-characteristic might be self-intersecting in the 3D embedding space (Fig 3).

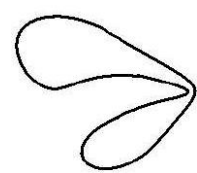

a)

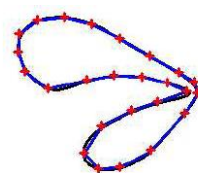

b)

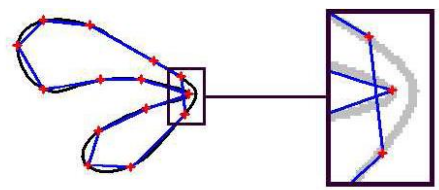

c)

d)

FIGURE 3. a) a simple closed curve with the topology of a circle. b) One example of a polyhedral decomposition of the curve using 25 vertices and edges. The corresponding Euler-characteristic $\chi=v-e=0$ is that of a circle. c) Another discretization of the same curve using 14 edges and vertices. Note that the Euler-characteristic is still that of a circle $\chi=0$, even though the discrete representation of the curve self-intersects in the $2 \mathrm{D}$ embedding space. d) Close-up.

\section{C - Topological Defects, Duality Foreground/Background}

In this chapter, we call topological defect any deviation from spherical topology: cavities, disconnected components, or handles. We note that for each defect present in a geometric entity, referred to as the foreground object, there exists a corresponding defect in the background (i.e. the embedding space): a disconnected foreground component can be interpreted as a background cavity; a foreground cavity is a disconnected background component; and a handle in a foreground component defines another handle in the background component. This foreground/background duality is of crucial importance for all retrospective topology correction techniques, as it provides a dual methodology to correct a topological defect. For in- 
stance, the presence of a handle in an object could be corrected by either cutting the handle in the foreground object, or cutting the corresponding handle in the background object. Cutting the background handle can be interpreted as filling the corresponding foreground hole (Fig. 4-b,c).

\subsection{Topology and Discrete Imaging}

In order to apply topological concepts to a discrete framework and define the topology type (i.e. homotopy type) of digital segmentations, the notion of continuity must be adapted to discrete spaces and objects, such as 3D image grids and triangulations. This is obtained by replacing the notion of continuity with the weaker notion of connectivity. We describe how topological notions can be adapted to the two most common data structures used in medical imaging: 3D data structures and surfaces.

A - Digital Topology

Digital topology provides an elegant framework, which translates the continuous concepts of topology to discrete images. In this theory, binary images inherit a precise topological meaning. In particular, the concept of homotopic deformation, which is required to assign a topological type to a digital object, is clearly defined through the notion of simple point. An extensive discussion of these concepts can be found in [36]. In this section, some basic notions of digital topology are presented. All definitions are from the work of G. Bertrand, which we refer to for more details [7].

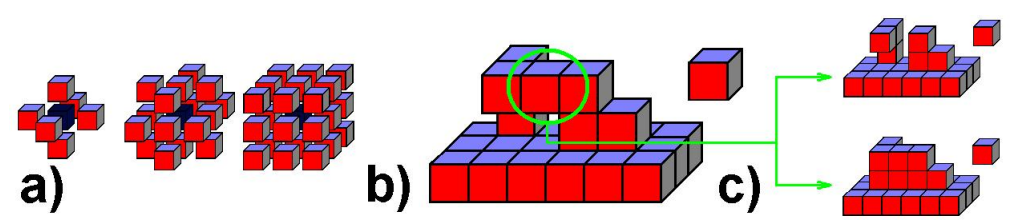

FIGURE 4. a) 6-, 18- and 26-connectivity. b) The circled voxel is a non-simple point c) Several dual topological corrections are possible: either cutting the handle (top) or filling the hole (bottom).

In the digital topology framework, a 3D image $I$ is interpreted as a graph. The vertices of the graph are the digital points (i.e. the voxels) and the edges are defined through neighborhood relations between points (i.e. the connectivity). A 3D binary digital image $I$ is composed of a foreground object $X$ and its inverse, the complement $\bar{X}$. We first need to define the concept of connectivity, which specifies the condition of adjacency that two points must fulfill to be regarded as connected. Three types of connectivity are commonly used in 3D: 6-, 18- and 26-connectivity. Two voxels are 6adjacent if they share a face, 18-adjacent if they share at least an edge and 26-adjacent if they share at least a corner (Fig. 4-a). In order to avoid topological paradoxes, different connectivities, $n$ and $\bar{n}$, must be used for one 
digital object $X$ and its complement $\bar{X}$. This leaves us with four pairs of compatible connectivities: $(6,26),(6,18),(26,6)$ and $(18,6)$. One important consequence of the previous requirement is that digital topology does not provide a consistent framework for multi-label images. No compatible connectivities can be chosen for neighboring components of the same object. Therefore, digital topology is strictly limited to binary images.

We now come to the definition of a simple point. This concept is central to most digital segmentation methods that integrate topological constraints [3, $26,32,36,42,44,49]$.

Definition 1.1 Simple point $A$ point of a binary object is simple if it can be added or removed without changing the topology of both the object and the background, that is, without changing the number of connected components, cavities and handles of both $X$ and $\bar{X}$ (Fig. 4-b,c).

A simple point is easily characterized by two topological numbers with respect to the digital object $(X, \bar{X})$ and a consistent connectivity pair $(n, \bar{n})$. These numbers, denoted $T_{n}(\mathbf{x}, X)$ and $T_{\bar{n}}(\mathbf{x}, \bar{X})$, were introduced by $\mathrm{G}$. Bertrand in [1] as an elegant way to classify the topology type of a given voxel. The values of $T_{n}(\mathbf{x}, X)$ and $T_{\bar{n}}(\mathbf{x}, \bar{X})$ characterize isolated, interior and border points as well as different kinds of junctions. In particular, a point is simple if and only if $T_{n}(\mathbf{x}, X)=T_{\bar{n}}(\mathbf{x}, \bar{X})=1$. Their efficient computation, which only involves the 26-neighborhood, is described in [6].

The definition of a discrete homotopy follows from the concept of simple point.

Definition 1.2 Homotopic deformation We define a homotopic deformation of an object $X$ as a sequence of deletions or additions of simple points.

Finally, two objects $X$ and $Y$ share the same homotopy type if there exists a sequence of transformations $X_{0} \ldots X_{k}$ and a sequence of points $\mathbf{x}_{1} \ldots \mathbf{x}_{k}$, such that $X_{0}=X$ and $X_{i-1}=X_{i} \bigcup\left\{\mathbf{x}_{i}\right\}$ or $X_{i-1}=X_{i} \backslash\left\{\mathbf{x}_{i}\right\}$ and the point $\mathbf{x}_{i}$ is simple relative to $X_{i}$ for $i=1, \ldots, k$.

To be complete, we also mention some recent research in digital topology, such as the concept of multisimple point in $[45,44]$ to characterize the modification of the genus, a novel characterization of homeomorphic deformation in [4], as well as some octree grid topology concept in [1].

$B$ - Surfaces in Discrete Imaging

We now turn to the translation of continuous topological concepts to discrete surface representations. There are essentially two ways of representing a surface in discrete imaging. Surfaces can be either represented explicitly, by using a parameterized polygonal decomposition, or implicitly as the level set of some function defined in the 3D embedding space. Each type of representation has advantages and disadvantages, and has been extensively used for the purpose of medical image segmentation [11, 13, 21, 23, 35, 37, $57,58,59]$. 

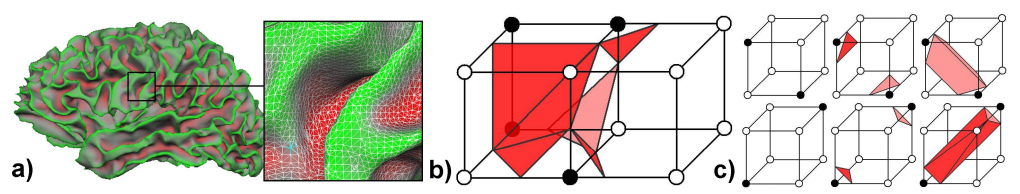

FIGURE 5. a) A triangulated cortical surface b) Tiling inconscistency in isosurface extraction c) Different tessellations corresponding to different topology types.

\section{B.1 - Explicit Representations}

An explicit representation models a surface by a set of vertices, edges, and faces, associated with a chosen parameterization of each face. The set of vertices, edges, and faces composes the polyhedral representation of the surface. The parameterization of the faces determines the exact geometry of the surface. For instance, tessellations correspond to linear parameterizations of each face, while splines use higher-order approximations. Triangulations are a special kind of tessellation, in which each face is a triangle (Fig. 5-a).

The topological invariance of the Euler-characteristic implies that explicit models unambiguously encode the intrinsic topology of the surfaces. However, there is no guarantee that the surface representation is not selfintersecting. As previously mentioned, the topological equivalence defining the intrinsic topology of a geometric entity ignores the embedding space. Consequently, additional precautions must be taken in order to ensure that the discretization of a surface does not generate self-intersections. The self-intersection problem is important when the surfaces are iteratively deformed in order to match a targeted structure (i.e. theory of active contours).

Finally, we note that explicit representations can approximate surfaces at any level of precision, by using more refined meshes. Contrary to the theory of digital topology that constitutes a discrete approximation of the continuous space, and is therefore limited by the resolution of the 3D digital images, explicit representations can accurately approximate any surface by using high-resolution meshes.

\section{B.2 - Implicit Representations}

Implicit models encode the surface of interest $\mathcal{C}$ as the level set of a higherdimensional function $\phi$ defined in the embedding space $\mathbb{R}^{3}$. The function $\phi$, defined on a $3 \mathrm{D}$ voxel grid, is usually the signed distance function of the surface with the contour being the zero level set of $\phi$ : $\mathcal{C}=\phi^{-1}(0)$.

This type of representation has several advantages. First, no explicit representation and no parameterization are required. In the theory of active contours, this has proven to be a huge advantage as implicit representations can naturally change topology during the deformation of the model. Selfintersections, which are costly to prevent in parametric deformable models, are avoided and topological changes are automated. In addition, many fun- 
damental properties of the surface $\mathcal{C}$, such as its normal or its curvature, are easily computed from the level set function $\phi$.

However, these models can only represent manifolds of codimension one without borders, i.e. closed surfaces in $\mathbb{R}^{3}$. For the purpose of segmenting anatomical structures, the use of such representations is not a limitation. Another - more subtle - drawback of implicit representations is that, even though level sets achieve sub-voxel accuracy, the exact location of the contour depends on the image resolution. For instance, in the case of two adjacent banks of a sulcus that are closer than the resolution of the underlying voxel grid (or physically touching), the finite image resolution and the topological constraint necessitate some voxels to be labeled as outside voxels (ideally, these voxels should be the ones belonging to CSF), thus imposing a constraint on the location and accuracy of the surface model (some recent methods to alleviate this limitation have been proposed in $[2,27]$ ).

So far, we have not specified how implicit representations can ensure that the topology of the encoded surface is the correct one. Since implicit representations make use of the underlying $3 \mathrm{D}$ voxel grid (through a signed distance function $\phi$ ) to encode the contour of interest, digital topology (sec. 2.2-A) can be used to specify the topology of the contour [28]. The foreground object $X$ is simply defined as the set of negative grid points (i.e. $X=\left\{\mathbf{x} \in \mathbb{R}^{3} \mid \phi(\mathbf{x}) \leq 0\right\}$ ), and the background object $\bar{X}$ as the set of strictly positive grid points (i.e. $\bar{X}=\left\{\mathbf{x} \in \mathbb{R}^{3} \mid \phi(\mathbf{x})>0\right\}$ ). Then, given a choice of compatible connectivities, the topology of the contour is determined unambiguously.

\section{C - From Images to Surfaces: Isocontour Extraction}

In the previous section, we described the manner in which topology can be adapted to the two most common data structures used in medical imaging. The ability to go from one representation to the next arises as a difficulty. Although it is possible to generate triangulations from 3D binary digital segmentations, such that the resulting topology of the surfaces is consistent with the choice of digital topology, it is not always possible to produce a digital binary representation, whose topology is similar to that of a given triangulation: digital topology constitutes a discrete approximation of the continuous space at a finite resolution, while triangulations approximate continuous surfaces at any level of precision.

The marching cubes (MC) algorithm was first introduced by Lorensen and Cline in 1987 [34] as a way to generate a polygonal decomposition (e.g. a triangulation) from a scalar field sampled on a rectilinear grid (e.g. an implicit representation). Given an isovalue, the MC algorithm quickly extracts a representation of the isosurface of the scalar field. The MC algorithm first partitions the data into a set of cubic (or rectilinear) cells, the cell vertices being the grid points. Based on the relative polarity of their scalar value (above or below the isovalue), each vertex is assigned a binary label, which indicates whether the grid point is inside or outside the isosurface. Then, 
each cubic cell is processed sequentially. Patches (i.e. sets of triangles) that approximate the isosurface (based on tri-linear interpolation) are produced within each cube, and the polygon patches are naturally joined together to form the final isosurface representation.

Unfortunately, the standard marching squares or marching cubes algorithm does not generate topologically consistent tessellations, since the resulting tessellations may contain tiling and topological inconsistencies (Fig. 5-b). In order to alleviate this problem, Han et al. [28] have designed a modified connectivity-consistent marching contour algorithm, by building a specialized case table for each type of digital topology (Fig. 5-c). Extensive discussion of isocontour extraction algorithms can be found in the thesis of Han [25]. Note also some new research directions such as [1].

\section{State of the Art in Segmentation under Topological Constraints}

As noted previously, methods for producing topologically-correct segmentations can be broadly divided into two categories. A first set of approaches directly incorporates topological constraints into the segmentation process, while another set aims at correcting retrospectively the spherical topology of an already segmented image.

\subsection{Topologically-Constrained Segmentations}

The topology-enforcing techniques proceed by iteratively deforming a model of known topology onto a targeted structure, while preserving its topology. Several techniques have been used for the segmentation of anatomical structures, with the topological constraint taking different forms depending on the chosen method.

\section{A - Active Contours}

Depending on the representation, two different implementations are usually encountered. One encodes the manifold of interest with an explicit representation using a Lagrangian formulation [52], while another implicitly represents the contour as the level set of a function defined on higherdimensional manifold in an Eulerian formulation [9, 40].

- Parameterized models maintain an explicit representation of the contour and preserve its initial intrinsic topology. Any level of accuracy can be achieved by using more refined meshes. However, the preservation of the whole topology also requires the prevention of self-intersections, which proves to be computationally intensive and requires elaborate methods to detect and prevent surface intersection during the evolution. Also, note that the preservation of the initial topology is often a strong limitation to most 
explicit models, since a fully automatic and efficient handling of topology changes using explicit models remains an open issue [14, 15, 33, 41, 38, 39]. - The ability to automatically handle topological changes is a long acknowledged advantage of the level set method over explicit deformable models, but may not be desirable in some applications where some prior knowledge of the target topology is available. This is typically the case in biomedical image segmentation, where the topology of the organs and their mutual topological relations is prescribed by anatomical knowledge (in the absence of gross pathology). In order to overcome this problem, a topologypreserving variant of the level set method has been proposed in [28]. This method is based on the theory of digital topology and uses the underlying embedding space to constrain the topology of the interior of the level set. However, the strict topology preservation necessitates an initialization of the active contour that is close to its final configuration in order to avoid topological barriers that can easily generate large geometrical errors. In the case of complex structures, like the cortical surface, such initialization proves to be extremely difficult [26].

Recently, another variant was proposed to exert a more subtle topological control on a level set evolution by allowing connected components to merge, split or vanish without changing the genus of the active contours [44]. While the original level set model does not provide any topological control, topology-preserving level sets enforce a strong constraint that is often too restrictive. This framework establishes a trade-off between the two models. It offers a more fine-grained topological control that alleviates many problems of methods that enforce a strong topological constraint (e.g. sensitivity to initialization and noise, simultaneous evolution of multiple components and speed of convergence).

\section{B - Digital Homotopic Deformations}

Similar to active contour models, digital approaches [8, 3, 5, 36, 42] deform an initial region with a known given topology (typically a single voxel carrying a spherical topology), by addition/deletion of points, minimizing a global energy functional while preserving the correct digital topology. Regions are grown or shrunk by adding points that will not change the region topology. Most of these methods are based on the theory of digital topology and the notion of simple points.

\section{$C$ - Segmentation by Registration}

Some approaches have been proposed to match a template with a given topology onto a specified MRI image $[3,10,30]$. These methods have the strong advantage of being able to enforce complex topology in the segmentation process, and to encode the spatial relationships that exist in between structures $[4,5]$. Nevertheless, the design of elaborate templates that include several structures with the correct topology is challenging. 
D - Limitations of Topologically-Constrained Segmentations

All these methods have the advantage of allowing the user to specify the proper topology and not allowing it to change. In the case of segmentation by registration, full brain models containing several structures can be matched onto a targeted image. Unfortunately, these methods are highly sensitive with regard to their initialization, and accurate final configurations most often require an initialization of the models that is close to its final configuration. One of the main reasons is that the energy functionals driving the deformation are typically highly non-convex and the evolution is therefore easily trapped in local minima.

A significant drawback of topologically constrained evolution is that it can lead to large geometric errors, due to the topological constraint and the presence of topological barriers (constituted by sets of non-simple points in the case of digital segmentations, self-touching and frozen surface regions in active contours segmentations). This is the case for methods that aim at segmenting the cortex starting from one single object located deep inside the cortical surface. Large topological barriers are often generated during the template deformation leading to inaccurate final segmentations. This is mostly a result of the presence of noise in the image and of the fact that topologically constrained segmentation prevents the formation of cavities (easy to detect and suppress) as well as the formation of handles.

Finally, we note that digital methods, as well as implicit representations that use the $3 \mathrm{D}$ embedding space to encode the surface of interest, are constrained by the finite resolution of the $3 \mathrm{D}$ grid and may not be able to represent deep folds in the target structure. To solve this problem, Han et al. [27] have implemented a moving grid algorithm, which aims at optimally deforming the underlying 3D grid for accurate implicit representations. Let us also mention some recent octree-based topology-preserving geometric deformable model [2].

\subsection{Retrospective Topology Correction}

Recently, new approaches have been developed to retrospectively correct the topology of an already-segmented image. These techniques, which do not impose any topological constraints on the segmentation process, can focus on attaining more accurate models with few topological inconsistencies to be identified and corrected post-hoc. These methods can be divided into two main classes: volume-based methods that work directly on the volume lattice and correct the topology by addition/deletion of voxels $[26,32,46,49]$, and surface-based methods that aim at modifying the tessellation by locating and cutting handles [19, 24, 47, 48].

A - Volume-Based Approaches

Most volume-based approaches have been specifically designed to enforce the spherical topology of cortical surface. These methods identify the lo- 
cation of the topological defects present in the segmentation by building a graph encoding the connectivity of the segmentation; the topological defects present in the volume are then corrected by introducing some cuts in the connectivity graph (e.g. modifying the binary labels of some key voxels in the volume).

One of the most inspirational approaches in this domain is certainly the pioneering work of Shattuck and Leahy [49]. One drawback of their approach is that the "cuts", which are necessary to correct the topological defects, can only be oriented along the Cartesian axes and give rise to "unnatural" topological corrections. Their method is based on the theory of digital topology but is limited to 6-connectivity and has not been generalized for any other connectivity rule.

Han et al. developed an algorithm to correct the topology of a binary object under any digital connectivity [26]. They detect handles by graph analysis, using successive foreground and background morphological openings to iteratively break the potential topological defects at the smallest scales. In contrast to the approach of Shattuck and Leahy, "cuts" are not forced to be oriented along cardinal axes. However, topological corrections at a specific scale depend on the choice of filter, either foreground or background morphological filter, which fails to evaluate simultaneously the effect of two complementary dual solutions (i.e. cutting the handle or filling the corresponding hole) on the corrected segmentation.

Kriegeskorte and Goeble proposed a region growing method prioritized by the distance-to-surface of the voxels in order to force the cuts to be located at the thinnest part of each topological defect [32]. The same process is applied to the inverse object, offering an alternative solution to each cut. An empirical cost is then assigned to each solution and the final decision is the one minimizing the global cost function.

While these methods can be effective, they cannot be used to correct the topology of arbitrary segmentations, as they make assumptions regarding the topology of the initial input image. Most frequently, fully-connected volumes are assumed and cavities are supposed to be removed as a preprocessing step. In addition, they do not integrate any statistical or geometric information into the topology correction process. To alleviate these limitations, Ségonne et al. [46, 45] propose a topology correction approach that is phrased within the theory of Bayesian parameter estimation and integrates statistical information into the topology correction process. In addition, no assumption is made about the topology of the initial input images.

\section{$B$ - Surface-Based Approaches}

Approaches of the other type operate directly on the triangulated surface mesh. Topological defects are located either as intersections of wavefronts propagating on the tessellation $[24,29]$ or as non-homeomorphic regions between the initial triangulation and a sphere [19, 47, 48].

In $[24,29]$, a randomly selected vertex is used to initialize a region 
growing algorithm, which detects loops (i.e. topological defects) in the triangulation where wavefronts meet. Topological corrections are obtained through the use of opening operators on the triangle mesh, resulting in a fast method that depends on the initially selected vertex. In a similar work, Jaume [29] identifies minimal loops in the volume by wavefront propagation. This method assumes that the initial triangulation was generated through the use of a topologically-consistent algorithm. The minimal loops are then used to identify non-simple voxels in the volume, which are subsequently deleted. Again, this approach orients the "cuts" along the Cartesian axes and generates "unnatural" topological corrections. In addition, both methods ignore all additional information, such as the underlying intensity profile or the expected local curvature, and the resulting topological corrections might not be accurate.

Fischl et al. [19] proposed an automated procedure to locate topological defects by homeomorphically mapping the initial triangulation onto a sphere. Topological defects are identified as regions in which the homeomorphic mapping is broken and a greedy algorithm is used to retessellate incorrect patches, constraining the topology on the sphere $\mathcal{S}$ while preserving geometric accuracy by a maximum likelihood optimization. Unfortunately, this method relies on a greedy algorithm and the reconstructed final surface might be inaccurate. In addition, even though the final intrinsic topology will be the correct one, the proposed method cannot guarantee that final surface will not self-intersect.

Recently, Ségonne et al. proposed a methodology $[47,48]$ that alleviates most limitations of previous approaches, and is able to generate accurate topological corrections by integrating statistical and geometric information into the topology correction process while guaranteeing that the final surface will not self-intersect. Non-separating loops locate handles present in the volume, and produce topologically-corrected candidate solutions by discarding the faces that form the loops and by sealing the open mesh. The accuracy of each candidate solution is then maximized by active contour optimization. Finally, randomly-generated candidate solutions are selected based on their goodness of fit in a Bayesian framework.

\section{C - Limitations of Retrospective Topology Correction Algorithms}

Most of these methods assume that the topological defects in the segmentation are located at the thinnest parts of the segmented volume and aim to correct the topology by minimally modifying the volume or tessellation $[24,26,29,32,49]$. Although this will often lead to accurate results, due to the accuracy of initial segmentations, topological corrections may not be optimal: additional information, such as the expected local curvature or the local intensity distribution, may lead to different corrections (i.e. hopefully comparable to the ones a trained operator would make). In addition, digital methods often suffer from the finite resolution of the digital grids, posing a problem to the accurate location of the potential 
cuts.

Few methods have been proposed to integrate into the segmentation process some additional information, such as intensity or curvature $[46,47,48]$. Although the method introduced in [46] has the advantage of correcting the topology of any initial segmentation without making any assumption on the initial connectedness of the segmentation, it suffers from the same limitations as most digital methods (i.e. the accurate location of the potential cuts). Also, as most retrospective topology correction methods it only evaluates a small number of potential topological corrections per defect (i.e. only two dual corrections), consequently failing often to produce optimal solutions. To our knowledge, only the approach developed in [48] has been proposed thus far to generate multiple solutions and explore the full space of potential solutions in order to select the best correction of a topological defect.

\section{Conclusion}

In this chapter, we covered methods for integrating topological constraints into segmentation procedures in order to generate geometrically accurate and topologically correct models. We introduced some elementary but essential notions of topology (sec. 2.1), such as the concepts of homeomorphism and homotopy that are necessary to characterize the topological type of a geometric object. We have clearly distinguished the intrinsic topology of an object from its homotopy type (sec. 2.1). Also, we have emphasized the connections linking topology and differential geometry, such as the crucial notion of the Euler-characteristic of a surface.

The adaptation of the continuous concepts of topology into a discrete framework that is practical to the segmentation of medical images proves to be challenging. However, we have shown that topologically-consistent frameworks can be constructed by replacing the notion of continuity by the weaker notion of connectivity (sec. 2.2), using concepts from the theory of digital topology is (sec. 2.2-A). In particular, we have introduced the important concepts of simple point and topological numbers, and defined the discrete equivalent of homotopic deformations based on the notion of simple point. We have also presented isocontour extraction techniques (sec. 2.2-C).

Finally, we described current state-of-the-art segmentation of images under topological constraints and detailed the limitations of existing approaches (sec. 3). Among state-of-art techniques, retrospective methods (sec. 3.2) achieve overall better results than topologically constrained segmentation methods (sec. 3.1). In addition, techniques that integrate additional information, such as intensity or curvature, often lead to more accurate segmentations. However, segmentation under topological constraints remains a challenge with several promising research directions $[2,4,5,44$, 48]. 


\section{REFERENCES}

[1] Y. Bai, X. Han, and J. Prince. Octree-based topology-preserving isosurface simplification. In $M M B I A, 2006$.

[2] Y. Bai, X. Han, and J. Prince. Octree grid topology preserving geometric deformable models for $3 \mathrm{~d}$ medical image segmentation. In International Conference on Information Processing in Medical Imaging, 2007.

[3] P. Bazin and P. D.L. Topology preserving tissue classification with fast marching and topology templates. International Conference on Information Processing in Medical Imaging, pages 234-245, 2005.

[4] P. Bazin, L. Ellingsen, and D. Pham. Digital homeomorphisms in deformable registration. In International Conference on Information Processing in Medical Imaging, 2007.

[5] P. Bazin and D. Pham. Statistical and topological atlas based brain image segmentation. In Medical Image Computing and ComputerAssisted Intervention, 2007.

[6] G. Bertrand. A boolean characterization of three-dimensional simple points. Pattern Recognition Letters, 17:115-124, 1996.

[7] G. Bertrand and G. Malandain. A new characterization of threedimensional simple points. Patternb Recognition Letters, 2(15):169$175,1994$.

[8] S. Bischoff and L. Kobbelt. Isosurface reconstruction with topology control. Pacific Graphics Proceedings, pages 246-255, 2002.

[9] V. Caselles, R. Kimmel, and G. Sapiro. Geodesic active contours. The International Journal of Computer, 22(1):61-79, 1997.

[10] G. Christense, R. Rabbitt, and M. Miller. 3d brain mapping using a deformable neuroanatomy. Phys. Med. Biol., 39:609-618, 1994.

[11] A. Dale, B. Fischl, and S. M.I. Cortical surface-based analysis i: Segmentation and surface reconstruction. NeuroImage, 9:179-194, 1999.

[12] A. M. Dale and M. I. Sereno. Improved localization of cortical activity by combining eeg and meg with mri cortical surface reconstruction: A linear approach. Journal of Cognitive Neuroscience, 5(2):162-176, 1993.

[13] C. Davatzikos and R. Bryan. Using a deformable surface model to obtain a shape representation of the cortex. IEEE TMI, 15:758-795, 1996. 
[14] H. Delingette. General object reconstruction based on simplex meshes. The International Journal of Computer Vision, 32(2):111-146, 1999.

[15] H. Delingette and J. Montagnat. Shape and topology constraints on parametric active contours. Computer Vision and Image Understanding, 83(2):140-171, 2001.

[16] R. Desikan, F. Ségonne, and etal. A computer generated labeling system for subdividing the human cerebral cortex on mri scans into gyral based regions of interest. Human Brain Mapping, 2005.

[17] H. Drury, D. Van Essen, C. Anderson, C. Lee, T. Coogan, and J. Lewis. Computerized mappings of the cerebral cortex: A multiresolution flattening method and a surface-based coordinate system. J. Cogn. Neurosci, 8(1):1-28, 1996.

[18] B. Fischl and A. Dale. Measuring the thickness of the human cerebral cortex from magnetic resonnace images. Proceedings of the National Academy of Sciences, 97:11044-11049, 2000.

[19] B. Fischl, A. Liu, and A. Dale. Automated manifold surgery: Constructing geometrically accurate and topologically correct models of the human cerebral cortex. IEEE TMI, 20:70-80, 2001.

[20] B. Fischl, D. Salat, E. Busa, M. Albert, M. Dieterich, C. Haselgrove, A. Van der Kouwe, R. Killinay, D. Kennedy, S. Klaveness, A. Montillo, N. Makris, B. Rosen, and A. Dale. Whole brain segmentation: Automated labeling of neuroanatomical strucutres in the human brain. Neuron, 33:341-355, 2002.

[21] B. Fischl, M. Sereno, and A. Dale. Cortical surface-based analysis ii: Inflation, flattening, and a surface-based coordinate system. NeuroImage, 9:195-207, 1999.

[22] B. Fischl, M. Sereno, R. Tootell, and A. Dale. High-resolution intersubject averaging and a coordinate system for the cortical surface. Human Brain Mapping, 8:272-284, 1999.

[23] R. Goldenberg, R. Kimmel, E. Rivlin, and M. Rudzsky. Cortex segmentation: A fast variational geometric approach. IEEE TMI, 21(2):1544-1551, 2002.

[24] I. Guskov and Z. Wood. Topological noise removal. Graphics I proceedings, pages 19-26, 2001.

[25] X. Han. Anatomically Consistent Segmentation of Medical Imagery Using a Level Set Method and Digital Topology. PhD thesis, Baltimore, Maryland, October 2003. 
[26] X. Han, C. Xu, U. Braga-Neto, and J. Prince. Topology correction in brain cortex segmentation using a multiscale, graph-based approach. IEEE TMI, 21(2):109-121, 2001.

[27] X. Han, C. Xu, and J. Prince. A 2d moving grid geometric deformable model. IEEE Conf. on Comp. Vis. Patt. Recog., pages 153-160, 2003.

[28] X. Han, C. Xu, and J. Prince. A topology preserving level set method for geometric deformable models. IEEE Transactions on Pattern Analysis and Machine Intelligence, 25(6):755-768, 2003.

[29] S. Jaume. Topology Simplification Algorithm for the Segmentation of Medical Images. PhD thesis, University of Louvain (Belgium), Feb 2004 .

[30] B. Karaç and C. Davatzikos. Topology preservation and regularity in estimated deformation fields. International Conference on Information Processing in Medical Imaging, pages 426-437, 2003.

[31] R. Kikinis and etal. Temporal love sulco-gyral pattern anomalies in schizophrenia: An in vivo $\mathrm{mr}$ three-dimensional surface rendering study. Neuroscience Letters, 182:7-12, 1994.

[32] N. Kriegeskorte and R. Goeble. An efficient algorithm for topologically segmentation of the cortical sheet in anatomical $\mathrm{mr}$ volumes. NeuroImage, 14:329-346, 2001.

[33] J.-O. Lachaud and A. Montanvert. Deformable meshes with automated topology changes for coarse-to-fine 3D surface extraction. Medical Image Analysis, 3(2):187-207, 1999.

[34] W. Lorensen and H. Cline. Marching cubes: A high-resolution 3D surface reconstruction algorithm. ACM Computer Graphics, 21(4):163$170,1987$.

[35] D. MacDonald, N. Kabani, D. Avis, and A. Evens. Automated 3d extraction of inner and outer surfaces of cerebral cortex from mri. NeuroImage, 12:340-356, 2000.

[36] J.-F. Mangin, V. Frouin, I. Bloch, J. Regis, and J. Lopez-Krahe. From $3 \mathrm{~d}$ magnetic resonance images to structural representations of the cortex topography using topology preserving deformations. Journal of Mathematical Imaging and Vision, 5:297-318, 1995.

[37] T. McInerney and D. Terzopolos. Deformable models in medical image analysis: A survey. Medical Image Analysis, 1(2):91-108, 1996.

[38] T. McInerney and D. Terzopolos. Deformable models in medical image analysis: A survey, 1999 update. Handbook of Medical Image Processing, 1999. 
[39] T. McInerney and D. Terzopoulos. T-snakes: Topology adaptive snakes. Medical Image Analysis, 4:73-91, 2000.

[40] S. Osher and J. Sethian. Fronts propagating with curvature-dependent speed: Algorithms based on Hamilton-Jacobi formulations. Journal of Computational Physics, 79(1):12-49, 1988.

[41] J.-P. Pons and J.-D. Boissonnat. Delaunay deformable models: Topology-adaptive meshes based on the restricted delaunay triangulation. In Conference on Computer Vision and Pattern Recognition, 2007.

[42] F. Poupon, J.-F. Mangin, D. Hasboun, C. Poupon, I. Magnin, and V. Frouin. Multi-object deformable templates dedicated to the segmentation of brain deep structures. LNCS, 1496:1134-1143, 1998.

[43] D. Salat, R. Buckner, A. Snyder, D. Greve, R. Desikan, E. Busa, J. Morris, A. Dale, and B. Fischl. Thinning of the cerebral cortex in aging. Cerebral Cortex, 14(7):721-730, July 2004.

[44] F. Ségonne. Active contours under topology control - genus preserving level sets. The International Journal of Computer, 2007.

[45] F. Ségonne. Segmentation of Medical Images under Topological Constraints. PhD thesis, Massachusetts Institute of Technology, December 2005.

[46] F. Ségonne, E. Grimson, and B. Fischl. Topological correction of subcortical segmentation. In Proceedings of Medical Image Computing and Computer-Assited Intervention, volume 2879-2, pages 695-702, 2003.

[47] F. Ségonne, E. Grimson, and B. Fischl. A genetic algorithm for the topology correction of cortical surfaces. In Proceedings of Information Processing in Medical Imaging, LNCS, volume 3565, pages 393-405, 2005.

[48] F. Ségonne, J. Pacheco, and B. Fischl. A geometrically accurate topology-correction of cortical surfaces using nonseparating loops. TMI, 26(4):518-529, 2007.

[49] D. Shattuck and R. Leahy. Automated graph based analysis and correction of cortical volume topology. IEEE TMI, 20(11):1167-1177, 2001.

[50] J. Tanabe, D. Amend, N. Schuff, V. DiSclafani, F. Ezekiel, D. Norman, G. Fein, and M. Wiener. Tissue segmentation of the brain in alzeihmer's disease. J. Neuroradiol., 18:115-123, 1997. 
[51] X. Tao, X. Han, M. Rettmann, J. Prince, and C. Davatzikos. Statistical study on cortical sulci of human brains. Proceedings of Inf. Proc. in Med. Imag., pages 37-49, 2001.

[52] D. Terzopoulos, A. Witkin, and M. Kass. Constraints on Deformable Models: Recovering 3D shape and Nonrigid Motion. Artificial Intelligence, 36(1):91-123, 1988.

[53] P. Thompson, D. MacDonald, M. Mega, C. Holmes, A. Evans, and A. Toga. Detection and mapping of abnormal brain structure with a probabilistic atlas of cortical surfaces. J. Comput. Assist. Tomogr., 21(4):567-581, 1998.

[54] P. Thompson, J. Moussai, S. Zohoori, A. Goldkorn, A. Khan, M. Mega, G. Small, J. Cummings, and A. Toga. Cortical variability and asymmetry in normal aging and alzheimer's disease. Cerebral Cortex, 8(6):492-509, 1998.

[55] M. Vaillant and C. Davatzikos. Hierarchical matching of cortical features for deformable brain image registration. Proceedings of Inf. Proc. in Med. Imag., pages 182-195, 1999.

[56] D. Van Essen and H. Drury. Structural and functional analyses of human cerebral cortex using a surface-based atlas. Journal of Neuroscience, 17(18):7079-7102, 1997.

[57] C. Xu, D. Pham, and J. Prince. Medical image segmentation using deformable models. Handbook of Medical Imaging - Medical Image Processing and Analysis, 2:129-174, 2000.

[58] C. Xu, D. Pham, M. Rettmann, D. Yu, and J. Prince. Reconstruction of the human cerebral cortex from magnetic resonance images. IEEE TMI, 18:467-480, 1999.

[59] X. Zeng, L. Staib, R. Schultz, and J. Duncan. Segmentation and measurement of the cortex from $3 \mathrm{~d}$ mr images using coupled surfaces propagation. IEEE TMI, 18:100-111, 1999. 